\title{
Der Einfluss kinematischer Parameter auf den Energie- bedarf eines Regalbediengerätes
}

\author{
The Influence of Kinematic Parameters on the Energy Need of a Storage and \\ Retrieval Vehicle
}

\author{
Robert Schulz \\ Jörg Monecke \\ Hartmut Zadek \\ Lehrstuhl für Logistik \\ Institut für Logistik und Materialflusstechnik, Fakultät Maschinenbau \\ Otto-von-Guericke-Universität Magdeburg
}

B isher wurde noch nicht erforscht wie sich die Variation kinematischer Parameter konkret auf den Energiebedarf von Regalbediengeräten auswirkt. Dieser Beitrag untersucht daher die energetischen Folgen bei unterschiedlichen Geschwindigkeiten und Beschleunigungen der Antriebe eines Regalbediengerätes. Veränderte Geschwindigkeiten und Beschleunigungen beeinflussen jedoch auch die Fahr- und Hubzeiten des Regalbediengerätes. Daher wird ebenfalls eine Berechnungsmethode vorgestellt, mit der der optimale Startpunkt des Hubwerkes und die optimale Fahrgeschwindigkeit auf Basis der eingestellten kinematischen Parameter berechnet werden können.

[Schlüsselwörter: Regalbediengerät, Energiebedarf, Geschwindigkeit, Beschleunigung, Startpunkt]

$\mathbf{S}^{\text {o }}$ far it is not investigated how varied kinematic parameters specifically make an impact on the energy needs of storage and retrieval vehicles. Thus this paper describes how varied settings of the speeds and accelerations of the powertrains affect the energy need of a storage and retrieval vehicle. However, changed speeds and accelerations have an impact on the drive and lift times of the storage and retrieval vehicle as well. Hence, in addition a calculation method is presented with which the optimal lifting unit's starting point and optimal drive speed may be calculated on the basis of the set kinematic parameters.

[Keywords: storage and retrieval vehicle, energy need, speed, acceleration, starting point]

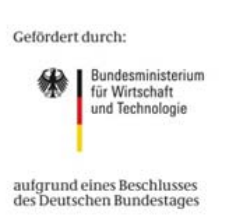

Förderhinweis: Dieser Beitrag entstand im Rahmen des IGFVorhabens Nr. 17389 BR ,Energieeffiziente Lagerstrategien und Lastverteilung“" der Forschungsvereinigung Bundesvereinigung Logistik e. V. Dieses Vorhaben wird von der AiF
über das Programm zur Förderung der industriellen Gemeinüber das Programm zur Förderung der industriellen Gemeinschaftsforschung- und Entwicklung (IGF) vom Bundesministerium fur Wirtschaft und Technologie aufgrund eines Be-
schlusses des deutschen Bundestages gefördert.

\section{EXISTIERENDE MASSNAHMEN ZUR REDUKTION DES} ENERGIEBEDARFS VON REGALBEDIENGERÄTEN

Bei hochautomatisierten Lägern haben die Bereiche Fördertechnik, Lager- und Kommissioniertechnik einen Anteil bis zu ca. 50\% an den Energiekosten (Heizungsund Belüftungstechnik ca. $35 \%$ und Beleuchtung ca. 15\%) [Kra08]. In der Intralogistik existieren bereits eine Reihe technologischer Maßnahmen, die zur Energieeffizienzsteigerung beitragen. Der Einsatz von energiesparenden Antrieben der Klasse IE2 und IE3 ist nicht für Regalbediengeräte (RBGs) geeignet, da ein hohes Anlaufmoment beim Anfahren überwunden werden muss, was durch die häufigen Anfahr- und Abbremsvorgänge insgesamt zu einem im Vergleich höheren Energiebedarf führt. Bei RBGs kommen jedoch andere Maßnahmen zum Einsatz, z. B. Leichtbauweise, rollwiderstandsarme Werkstoffkombinationen bei Rad und Schiene, Netzrückspeisungstechnik, Gleichstromzwischenkreiskopplung (Verwendung der beim Bremsen bzw. Senken des Fahr- bzw. Hubantriebs frei werdenden Energie für den jeweils anderen Antrieb), durchsatzoptimierter Fahrbetrieb, intelligente Fahrkurvenregelung (z. B. Vermeidung des gleichzeitigen Anfahrens zweier RBGs) oder Palettenheber mit Gegengewicht [Muh11]. Weiterhin ist in der Intralogistik allgemein bekannt, dass eine Reduzierung der Geschwindigkeit der Antriebe zu einer Verringerung des Energiebedarfs des Fördermittels führt. Welchen Einfluss die Parameter jedoch konkret ausüben, ist bisher nur in Ansätzen erforscht worden.

\section{DAS REGALBEDIENGERÄT AM INSTITUT FÜR LOGISTIK UND MATERIALFLUSSTECHNIK}

Dem Institut für Logistik und Materialflusstechnik (ILM) an der der Otto-von-Guericke-Universität Magdeburg steht ein $10 \mathrm{~m}$ langes und ca. 7,5 m hohes Automatisches Kleinteilelager (AKL) zur Verfügung (siehe Abbildung 1), mit dem die Energieeffizienz solcher Anlagen 
praxisnah erforscht werden soll. Die 400x600 mm großen Behälter werden mit einem RBG ein-, um- und ausgelagert. Im AKL am ILM (ILM-AKL) finden theoretisch 840 Behälter Platz (2 Regale mit jeweils 20 Fächern in horizontaler Richtung und 21 Fächern in vertikaler Richtung). Das Besondere an dieser Anlage ist, dass die Aufträge manuell eingegeben werden können. In der Praxis werden dagegen die Aufträge nur automatisch durch das Warehouse Management System vorgegeben. Weiterhin können beim ILM-AKL die kinematischen Parameter ausgehend von ihren Maximalwerten vor einem Experiment, d. h. einer Abfolge von Transportaufträgen, stufenlos eingestellt werden. Variiert werden können die Geschwindigkeiten sowie die positiven und negativen Beschleunigungen jedes einzelnen Antriebes, d. h. Fahrund Hubwerk des RBG sowie Gabel und Riemen des Lastaufnahmemittels. Weiterhin kann der Startzeitpunkt von Fahr- und Hubwerk in Abhängigkeit vom Fahrweg des jeweils anderen Antriebes (in \% des Fahr- bzw. Hubwegs) eingestellt werden.

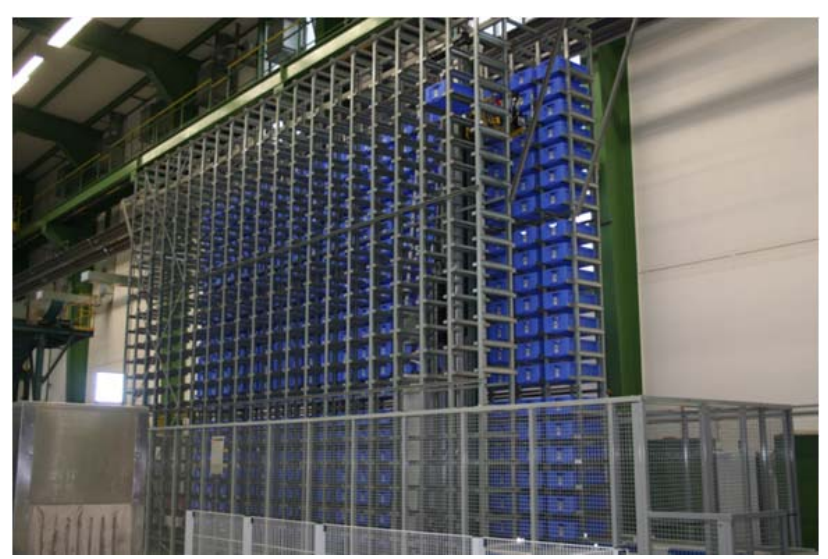

Abbildung 1. Das Automatische Kleinteilelager am Institut für Logistik und Materialflusstechnik an der Otto-von-Guericke-Universität Magdeburg

Die maximale Geschwindigkeit des Fahrwerkes beträgt $5,0 \mathrm{~m} / \mathrm{s}$ bei einer Beschleunigung von $3,0 \mathrm{~m} / \mathrm{s}^{2}$. Der Ruck (Begrenzung der Änderung der Beschleunigung über der Zeit) entspricht $6 \mathrm{~m} / \mathrm{s}^{3}$. Die durch das Fahrwerk bewegte Gesamtmasse entspricht ca. 1.996 kg. Das Hubwerk mit einer Eigenmasse von ca. $215 \mathrm{~kg}$ (180 kg Hubschlitten, $35 \mathrm{~kg}$ Lastaufnahmemittel) und einer maximalen Tragfähigkeit von $100 \mathrm{~kg}$ kann mit einer maximalen Geschwindigkeit von $\mathrm{v}_{\mathrm{HW}}=4,0 \mathrm{~m} / \mathrm{s}$ und einer maximalen Beschleunigung von $\mathrm{a}_{\mathrm{HW}}=4,0 \mathrm{~m} / \mathrm{s}^{2}\left(\mathrm{r}_{\mathrm{HW}}=8,0 \mathrm{~m} / \mathrm{s}^{3}\right)$ bewegt werden. Weiterhin verfügt die Anlage über die Möglichkeiten, bei Brems- und Absenkvorgängen entweder in das universiätsinterne Netz zurückzuspeisen oder über die Gleichstromzwischenkreiskopplung die frei werdende Energie dem jeweils anderen Antrieb zur Verfügung zu stellen.
Die Ergebnisse der Experimente liefern alle $20 \mathrm{~ms}$ Daten, die Informationen über die momentane Position und Leistungsaufnahme bzw. -rückspeisung aller Antriebe enthalten (siehe Abbildung 2). Somit können die zurückgelegten Wege des RBG, die Geschwindigkeiten, die Beschleunigungen und Elektroenergieaufnahmen bzw. rückspeisungen (in $\mathrm{kWs}$ ) über das komplette Experiment hinweg ermittelt werden.

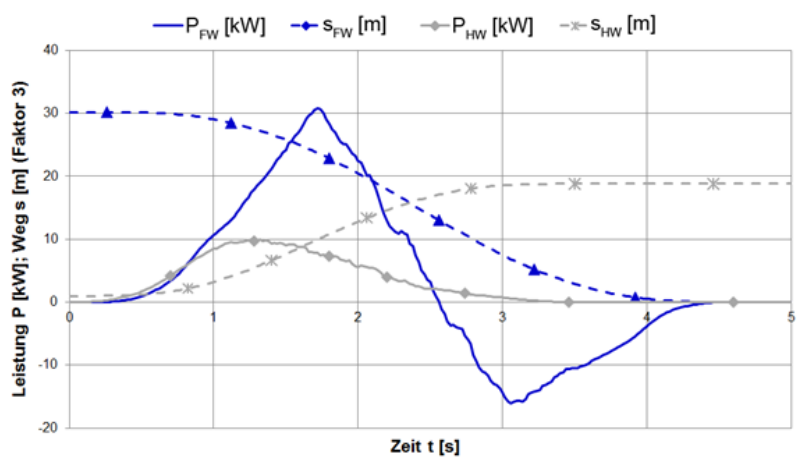

Abbildung 2. Beispiel-Datenaufzeichnung für eine Bewegung des Regalbediengerätes

\section{DER EINFLUSS DER GESCHWINDIGKEIT AUF DEN ENERGIEBEDARF DES FAHRWERKES}

Wie bereits in Abschnitt 1 beschrieben, werden die Geschwindigkeiten von RBGs bereits auf die Hälfte reduziert, wenn die Auftragslast unter ein bestimmtes Niveau sinkt. Wie sich der Energiebedarf jedoch konkret verändert, wenn die Maximalgeschwindigkeit variiert wird, ist bisher noch nicht erforscht und wird in diesem Kapitel aufgezeigt.

Durch eine Reduzierung der Geschwindigkeit ergeben sich zwei Effekte: Vorausgesetzt, dass die maximal eingestellte Geschwindigkeit erreicht wird, verkürzt sich zum einen als Resultat der Geschwindigkeitsverringerung die Anfahr- und Bremsphase. Für die Beschleunigung auf ein geringeres Geschwindigkeitsniveau wird weniger Energie benötigt. Zum anderen verlängert sich die Zeit, in der sich das RBG mit konstanter Geschwindigkeit bewegt. In der Phase der konstanten Geschwindigkeit muss bei einer geringeren Geschwindigkeit ein geringeres Widerstandsmoment überwunden werden, wodurch weniger Leistung aufgenommen werden muss. Die Auswirkungen von veränderten eingestellten Geschwindigkeiten des Fahrwerkes auf die Leistungsaufnahmen und -rückspeisungen, zeigt Abbildung 3 am Beispiel einer Fahrt, bei der der maximale Fahrweg bei maximalen Beschleunigungswerten zurückgelegt wird. In diesem Beispiel werden bei 4,0 m/s 27,2 kWs Elektroenergie vom Fahrwerk aufgenommen und 11,6 kWs zurückgespeist. Welche Energien bei verringerten Geschwindigkeiten aufgenommen und zurückgespeist werden sowie die zugehörigen Fahrzeiten für dieses Beispiel, zeigt Tabelle 1. 


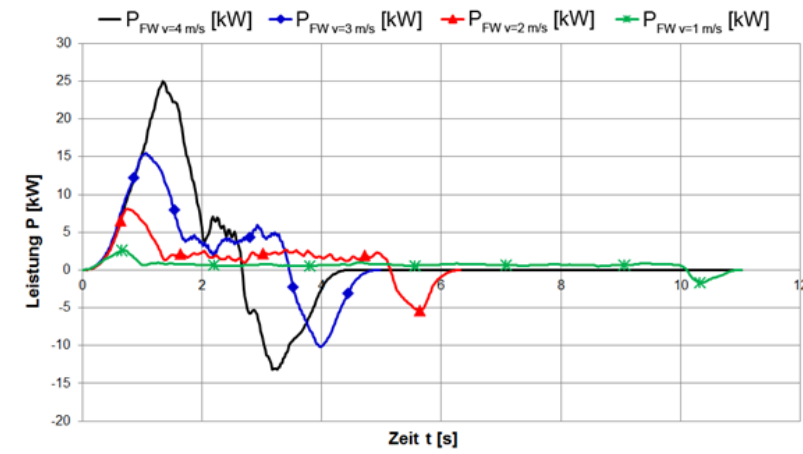

Abbildung 3. Der Einfluss veränderter Geschwindigkeiten auf die Leistungsaufnahme und -rückspeisung des Fahrwerkes

Diese Werte sind weiterhin vom zurückgelegten Weg abhängig. Diesen Zusammenhang zeigt Abbildung 4. Gut zu erkennen sind folgende Aspekte: In Abhängigkeit der eingestellten Geschwindigkeit stellen sich andere Beschleunigungszeiten und -wege ein. Bis zu bestimmten Fächern haben die Energiekurven bei unterschiedlichen Geschwindigkeiten somit einen identischen Verlauf, z. B. trennt sich die Kurve mit 2,0 m/s von den Kurven mit 3,0 $\mathrm{m} / \mathrm{s}$ und $4,0 \mathrm{~m} / \mathrm{s}$ bei ca. 4 Fächern $(2,0 \mathrm{~m})$. Ab diesem Weg geht die Kurve in eine Gerade mit konstanter Steigung über, d. h. dass ab diesem Weg die maximal eingestellte Geschwindigkeit erreicht wird und somit die gleiche Energie pro Wegeinheit für die Kompensierung der Verluste, z. B. um das Widerstandsmoment zu überwinden, benötigt wird (bei 2,0 m/s ca. $1,0 \mathrm{kWs} / \mathrm{m}$ ). Bis sich die Kurven voneinander trennen, wird die gleiche Geschwindigkeit bei gleichem Weg und gleicher Zeit erreicht. Bei der Kurve mit einer höheren eingestellten Geschwindigkeit ist die Beschleunigungsphase dann noch nicht abgeschlossen und die Geschwindigkeit nimmt weiter zu.

Eine verringerte Geschwindigkeit führt $\mathrm{zu}$ einer geringeren zurückgespeisten Energie, da diese von der erreichten Geschwindigkeit abhängt, von der heruntergebremst wird. Daher ist die rückgespeiste Energie auch konstant ab dem Weg, bei dem die eingestellte Geschwindigkeit erreicht wird. Auf der anderen Seite verlängert
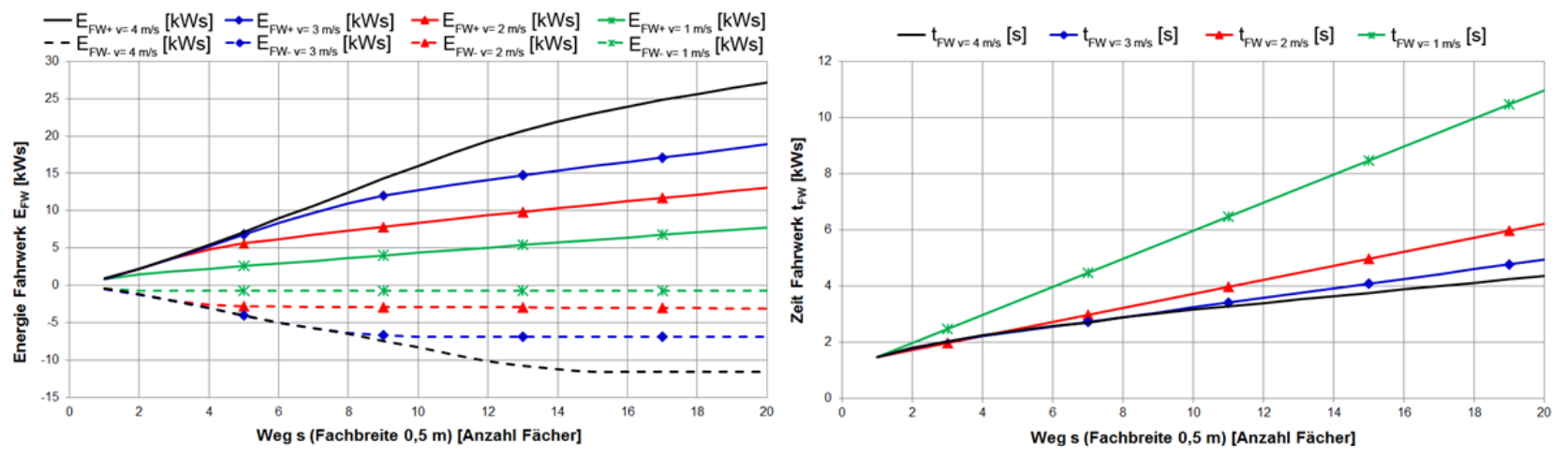

sich die Fahrzeit. Beispielsweise können bei maximalem Fahrweg und maximaler Beschleunigung ca. 36,5\% des Gesamtenergiebedarfs bei einer Reduzierung der eingestellten Geschwindigkeit von $4,0 \mathrm{~m} / \mathrm{s}$ auf $2,0 \mathrm{~m} / \mathrm{s}$ eingespart werden, wodurch sich jedoch die Fahrzeit um ca. 40,9\% erhöht (vgl. Tabelle 1).

Tabelle 1. Aufgenommene und zurückgespeiste Energien sowie Fahrzeiten bei unterschiedlichen Geschwindigkeiten am Beispiel des maximalen Fahrwegs

\begin{tabular}{|c|c|c|c|c|c|c|}
\hline $\begin{array}{c}\mathbf{V}_{\text {max }} \\
{[\mathbf{m} / \mathbf{s}]}\end{array}$ & $\begin{array}{c}\mathbf{E}_{\text {auf }} \\
{[\mathbf{k W s}]}\end{array}$ & $\begin{array}{c}\mathbf{E}_{\text {rück }} \\
{[\mathbf{k W s}]}\end{array}$ & $\begin{array}{c}\mathbf{E}_{\text {ges }} \\
{[\mathbf{k W s}]}\end{array}$ & $\begin{array}{c}\mathbf{t}_{\mathbf{F W}} \\
{[\mathbf{s}]}\end{array}$ & $\begin{array}{c}\Delta \mathbf{E}_{\text {ges }} \\
{[\% \mathbf{\%}]}\end{array}$ & $\Delta \mathbf{t}[\%]$ \\
\hline 4,0 & 27,2 & $-11,6$ & 15,6 & 4,4 & 0,0 & 0,0 \\
\hline 3,0 & 18,9 & $-6,9$ & 12,0 & 4,9 & $-23,1$ & $+11,4$ \\
\hline 2,0 & 13,0 & $-3,1$ & 9,9 & 6,2 & $-36,5$ & $+40,9$ \\
\hline 1,0 & 7,8 & $-0,7$ & 7,1 & 11,0 & $-54,5$ & $+150,0$ \\
\hline
\end{tabular}

\section{DER EINFLUSS DER BESCHLEUNIGUNG AUF DEN ENERGIEBEDARF DES FAHRWERKES}

Auch der Einfluss der Beschleunigung auf den Energiebedarf des Fahrwerkes ist bisher kaum erforscht. Oft werden in der Praxis RBGs nur mit geringeren Beschleunigungen betrieben um den Verschleiß zu reduzieren. Die Auswirkungen auf den Energiebedarf werden in diesem Abschnitt beschrieben.

Durch reduzierte Beschleunigungen ändern sich die Geschwindigkeitsverläufe. Unter der Voraussetzung, dass die maximal eingestellte Geschwindigkeit überhaupt bei einer geringeren Beschleunigung erreicht wird, verlängern sich zum einen die Anfahr- und Bremswege sowie -zeiten. Zum anderen verkürzen sich dadurch die Wege und Zeiten mit konstanter Geschwindigkeit. Die Energie, die zum Erreichen der eingestellten Geschwindigkeit benötigt wird, bleibt gleich. Für den Ausgleich der Verluste, die während der Phase der konstanten Geschwindigkeit kompensiert werden müssen, wird jedoch weniger Energie benötigt. In Summe sinkt dadurch der Energiebedarf bei geringeren Beschleunigungswerten, obwohl die Bewegung mit der gleichen eingestellten Geschwindigkeit durchgeführt wurde. Ein Beispiel dafür zeigt Abbildung 5, in der

Abbildung 4. Der Einfluss verschiedener Geschwindigkeiten auf den Energiebedarf und die -rückspeisung sowie Fahrzeiten in Abhängigkeit vom Fahrweg (1) 
der maximale Fahrweg mit 3,0 m/s und unterschiedlichen Beschleunigungen zurückgelegt wird.

Vorausgesetzt, dass die eingestellte Beschleunigung bei der jeweiligen Fahrt erreicht wird, verlängern sich bei reduzierten Beschleunigungen die Beschleunigungs- und Bremsphase und die Phase der konstanten Geschwindigkeit wird verkürzt. Durch diese Veränderungen im Vergleich zu einer Fahrt mit maximaler Beschleunigung erhöht sich die Fahrzeit. Da sich die Phase der konstanten Geschwindigkeit verkürzt, verringert sich der Energiebedarf für eine Fahrt bei reduzierter Beschleunigung.

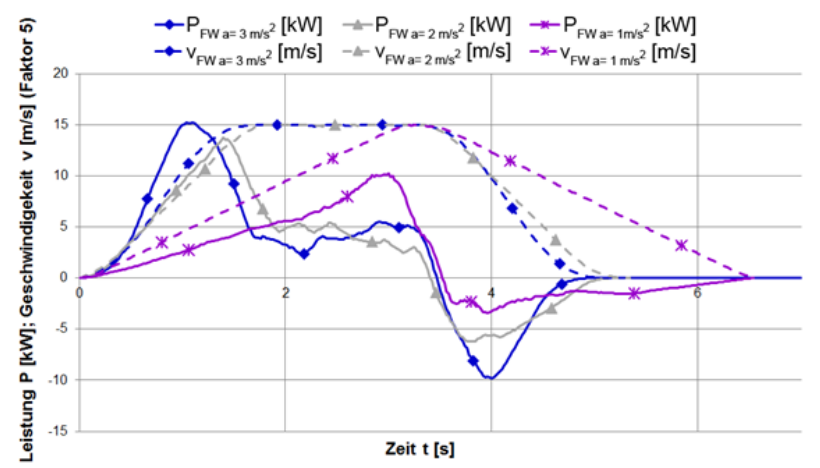

Abbildung 5. Der Einfluss veränderter Beschleunigungen auf die Leistungsaufnahme und -rückspeisung des Fahrwerkes

Tabelle 2 zeigt am Beispiel des Fahrwegs von 10 Fächern und einer eingestellten Geschwindigkeit von $3,0 \mathrm{~m} / \mathrm{s}$, welche Energien bei reduzierten Beschleunigungen aufgenommen und zurückgespeist werden sowie welche Zeit für die Fahrt benötigt wird.

Diese Werte sind ebenfalls vom zurückgelegten Weg abhängig, da bei reduzierten Beschleunigungen ggf. die eingestellte Geschwindigkeit nicht erreicht wird. Die aufgenommenen und zurückgespeisten Energien sowie Fahrzeiten zeigt Abbildung 6 für unterschiedliche Fahrwege bei einer eingestellten Geschwindigkeit von $3,0 \mathrm{~m} / \mathrm{s}$. Deutlich zu erkennen sind, ähnlich wie in Abbildung 4, die Fächer, ab denen bei der gewählten Einstellung die konstante Geschwindigkeit erreicht wird. Je geringer die maximale Beschleunigung eingestellt wird, desto später wird dieser Punkt erreicht. Bei der minimalen Beschleunigung von $1,0 \mathrm{~m} / \mathrm{s}^{2}$ wird die gewählte Geschwindigkeit erst bei maximalem Fahrweg erreicht (vgl. Abbildung 5). Wird die eingestellte Geschwindigkeit durch eine reduzierte Beschleunigung bei bestimmten Wegen nicht erreicht, sinken die aufgenommenen und zurückgespeisten Energien. Bei kleinen Beschleunigungen sinkt auch die zurückgespeiste Energie, weil auch beim Bremsen das Widerstandsmoment überwunden werden muss, das durch die verringerte negative Beschleunigung über einen längeren Zeitraum wirkt.

Tabelle 2. $\quad$ Aufgenommene und zurückgespeiste Energien sowie Fahrzeiten bei unterschiedlichen Beschleunigungen am Beispiel des Fahrwegs von 10 Fächern mit 3,0 m/s

\begin{tabular}{|c|c|c|c|c|c|c|}
\hline $\begin{array}{c}\mathbf{a}_{\mathbf{m a x}} \\
{\left[\mathbf{m} / \mathbf{s}^{\mathbf{2}}\right]}\end{array}$ & $\begin{array}{c}\mathbf{E}_{\text {auf }} \\
{[\mathbf{k W s}]}\end{array}$ & $\begin{array}{c}\mathbf{E}_{\text {rück }} \\
{[\mathbf{k W s}]}\end{array}$ & $\begin{array}{c}\mathbf{E}_{\text {ges }} \\
{[\mathbf{k W}]}\end{array}$ & $\begin{array}{c}\mathbf{t}_{\mathbf{F W}} \\
{[\mathbf{s}]}\end{array}$ & $\begin{array}{c}\Delta \mathbf{E}_{\text {ges }} \\
{[\mathbf{\%}]}\end{array}$ & $\begin{array}{c}\Delta \mathbf{t} \\
{[\%]}\end{array}$ \\
\hline 3,0 & 12,8 & $-6,9$ & 5,9 & 3,2 & 0,0 & 0,0 \\
\hline 2,0 & 11,7 & $-6,1$ & 5,6 & 3,5 & $-5,1$ & $+9,4$ \\
\hline 1,0 & 7,5 & $-2,8$ & 4,7 & 4,6 & $-20,3$ & $+43,8$ \\
\hline
\end{tabular}

Wie in Tabelle 2 ersichtlich, führen verringerte Beschleunigungen durch die Verkürzung der Phase konstanter Geschwindigkeit zu geringeren Gesamtenergiebedarfen bei gleichen zurückgelegten Wegen mit der gleichen eingestellten Geschwindigkeit. Auf der anderen Seite verlängern sich die Beschleunigungs- und Bremsphase, wodurch die Fahrzeit insgesamt verlängert wird. Aufgrund der längeren Beschleunigungs- und Bremswege (vgl. auch Abbildung 5) erhöht sich bei kleineren Beschleunigungen jedoch auch der Energiebedarf dieser Phasen und die Rückspeisung fällt geringer aus. Dadurch egalisiert sich beim Vergleich der Beschleunigung von $3,0 \mathrm{~m} / \mathrm{s}^{2}$ und $2,0 \mathrm{~m} / \mathrm{s}^{2}$ die Energieeinsparung ab einer Strecke von ca. 15 Fächern. Somit ist der Gesamtenergiebedarf ab diesem Weg bei einer Beschleunigung von $3,0 \mathrm{~m} / \mathrm{s}^{2}$ geringer.

Aus Abbildung 6 kann weiterhin geschlussfolgert werden, dass die Verzögerung vom Fahrwerk mit der maximalen negativen Beschleunigung durchgeführt werden sollte, um die Rückspeisung zu maximieren.

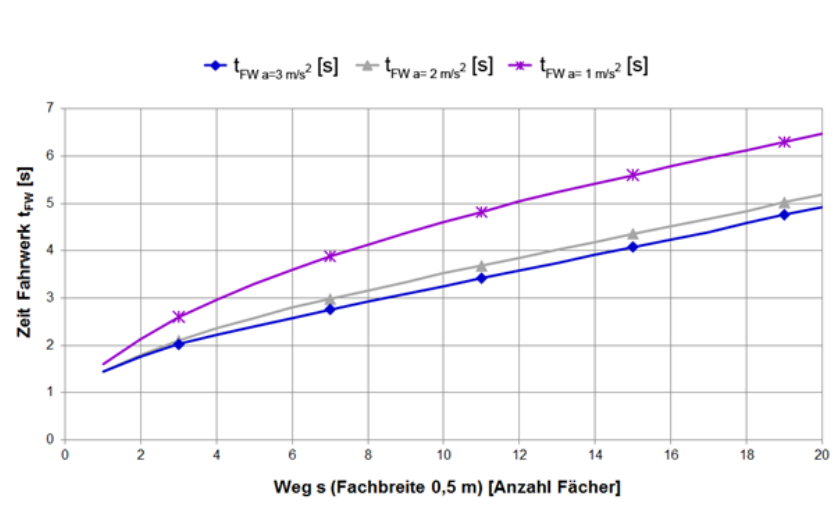

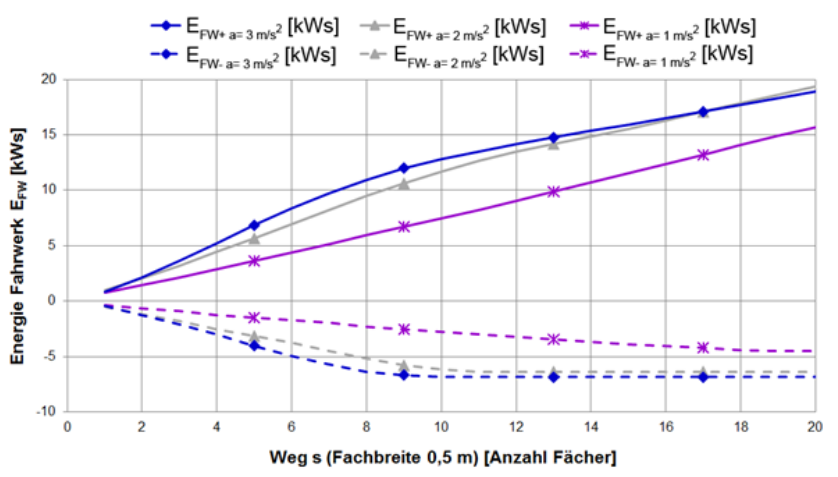

Abbildung 6. Der Einfluss verschiedener Beschleunigungen auf den Energiebedarf und die -rückspeisung sowie Fahrzeiten in Abhängigkeit vom Fahrweg 


\section{DER EINFLUSS DER GESCHWINDIGKEIT UND DER BESCHLEUNIGUNG AUF DEN ENERGIEBEDARF DES HUBWERKES}

Da in Abschnitt 3 und 4 die Auswirkungen von veränderten eingestellten Geschwindigkeiten und Beschleunigungen auf den Energiebedarf des Fahrwerkes des RBG betrachtet wurden, wird im Folgenden der Einfluss auf den Energiebedarf des Hubwerkes analysiert.

Die Auswirkungen reduzierter Geschwindigkeiten und Beschleunigungen auf die Bewegungsdauer sind beim Hubwerk analog zum Fahrwerk. Abbildung 7 zeigt am Beispiel des maximalen Hubwegs, welche Effekte sich aus verschiedenen eingestellten Geschwindigkeiten auf die Leistungsaufnahmen und -rückspeisungen des Hubwerkes ergeben. Je geringer die eingestellte Geschwindigkeit ist, desto geringer ist die maximale Leistungsaufnahme bzw. -rückspeisung. Weiterhin verkürzen sich wie beim Fahrwerk die Beschleunigungs- und Bremsphase, wohingegen sich die Phase mit konstanter Geschwindigkeit verlängert.

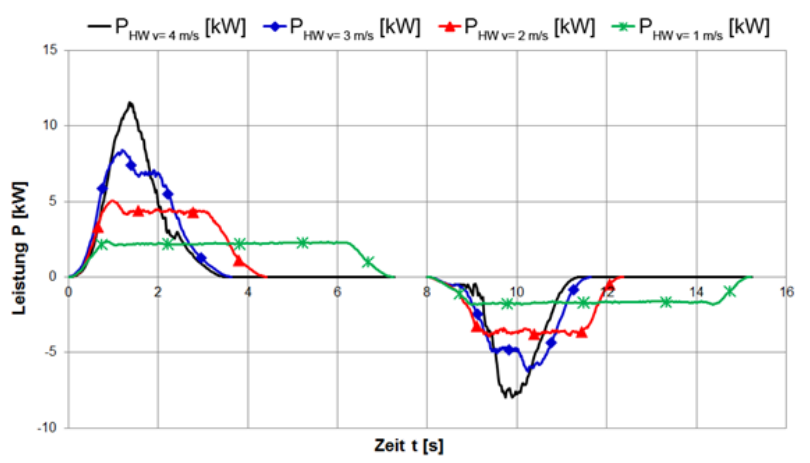

Abbildung 7. Der Einfluss veränderter Geschwindigkeiten auf die Leistungsaufnahme und -rückspeisung des Hubwerkes

Im Gegensatz zum Fahrwerk haben Geschwindigkeit und Beschleunigung keinen Einfluss auf den Gesamtenergiebedarf des Hubwerkes, weil die potentielle Energie nicht von diesen Parametern abhängt. Hinzu kommt lediglich ein Wirkungsgrad $\eta$ von ca. 90\% für die Hubbewegung $\left(\mathrm{E}_{\mathrm{pot}}=\mathrm{m} \cdot \mathrm{g} \cdot \Delta \mathrm{h} / \eta\right)$. Dies ist in Abbildung 7 anhand der gleichen Flächeninhalte unter den Kurven ersichtlich.

Die entsprechenden Daten zu den aufgenommen und zurückgespeisten Energien sowie die Zeiten, die für die Hubbewegung benötigt werden, enthält Tabelle 3. Die Abweichungen der Werte voneinander sind auf Messungenauigkeiten zurückzuführen. Es ist ersichtlich, dass die Geschwindigkeit keinen signifikanten Einfluss auf den Energiebedarf des Hubwerkes hat. Eine Verringerung der Geschwindigkeit führt dagegen zu einer Erhöhung der Hubzeiten, z. B. um 30,3\% bei einer Reduzierung von $4,0 \mathrm{~m} / \mathrm{s}$ auf $2,0 \mathrm{~m} / \mathrm{s}$.

Da Geschwindigkeit und Beschleunigung keinen Einfluss auf den Energiebedarf des Hubwerkes haben, ist es aus energetischer Sicht nicht sinnvoll, diese kinematischen Parameter zu reduzieren. Eine Verringerung der Beschleunigung könnte jedoch zur Reduzierung des Verschleißes des Hubwerkes zweckmäßig sein. Einige Firmen nutzen dies bereits bei ihren Anlagen (sowohl Fahrals auch Hubwerk). Allerdings sind auch infolgedessen Einbußen bei der Durchsatzleistung die Folge, da sich die Hub- und Senkzeiten erhöhen.

Tabelle 3. Aufgenommene und zurückgespeiste Energien sowie Hubzeiten bei unterschiedlichen Geschwindigkeiten am Beispiel des maximalen Hub- bzw. Senkweges

\begin{tabular}{|c|c|c|c|c|}
\hline $\mathbf{v}_{\mathbf{m a x}}[\mathbf{m} / \mathbf{s}]$ & $\mathbf{E}_{\text {Hub }}[\mathbf{k W s}]$ & $\mathbf{E}_{\text {Senk }}[\mathbf{k W s}]$ & $\mathbf{t}_{\mathbf{H W}}[\mathbf{s}]$ & $\boldsymbol{\Delta t}[\mathbf{\%}]$ \\
\hline 4,0 & 14,9 & $-10,0$ & 3,3 & 0,0 \\
\hline 3,0 & 14,6 & $-9,9$ & 3,5 & $+6,1$ \\
\hline 2,0 & 14,7 & $-10,2$ & 4,3 & $+30,3$ \\
\hline 1,0 & 14,0 & $-10,5$ & 7,1 & $+115,2$ \\
\hline
\end{tabular}

\section{OPTIMALER STARTPUNKT DES HUBWERKES UND OPTIMALE GESCHWINDIGKEIT DES FAHRWERKES}

Um einen möglichst energieeffizienten Betrieb eines RBG zu gewährleisten, sollten nicht nur die Geschwindigkeiten und Beschleunigungen angepasst werden, sondern auch der Startzeitpunkt des Hubwerkes so gewählt werden, dass möglichst viel der vom Fahrwerk zurückgespeisten Energie für die Hubbewegung genutzt wird. Erfolgt eine Senkbewegung, sollten beide Antriebe gleichzeitig starten, damit das Fahrwerk durch die Gleichstromzwischenkreiskopplung die freiwerdende Energie für die Beschleunigungsphase nutzen kann. Energieoptimal ist es, die Hubbewegung dann zu starten, wenn die Bremsbewegung des Fahrwerkes beginnt. Dadurch könnte sich gegebenenfalls die Arbeitsspielzeit verlängern.

Falls eine Verlängerung der Arbeitsspielzeit aufgrund der Durchsatzanforderungen nicht in Frage kommt, besteht die Möglichkeit, den Startpunkt des Hubwerkes so einzustellen, dass die Gesamtarbeitsspielzeit nicht verlängert wird. Hierfür existieren wiederum zwei Alternativen: Wenn die Bewegung des Fahrwerkes für die Arbeitsspielzeit maßgebend ist, kann der Startzeitpunkt des Hubwerkes so gewählt werden, dass die Hubbewegung erst dann startet sobald das Fahrwerk beginnt zu bremsen bzw. wenn die Hubbewegung länger dauert als die Bremsbewegung des Fahrwerkes, kann die Einstellung so gewählt werden, dass beide Bewegungen gleichzeitig enden. So wird der größte Teil der Bremsenergie des Fahrwerkes für die Hubbewegung genutzt. Eine Veränderung der Geschwindigkeit oder Beschleunigung des Hubwerkes ist wie in Abschnitt 5 beschrieben aus energetischer Sicht nicht sinnvoll. Somit sollten die Hubbewegungen mit den eingestellten Standardwerten durchgeführt werden. Ist jedoch die Hubzeit geringer als die Fahrzeit, sollte die Geschwindigkeit des Fahrwerkes so reguliert werden, dass die Antriebe gleichzeitig ankommen. Diese drei Fälle 
werden im Folgenden diskutiert. Beim ILM-AKL kann der Startpunkt der Antriebe in Abhängigkeit des zurückgelegten Weges des jeweils anderen Antriebs (in \%) eingestellt werden. Deshalb beziehen sich die folgenden Angaben immer auf den Weg.

\subsection{STARTPUNKT DES HUBWERKES IM ENERGIEEFFIZIENTEN BETRIEB}

Wie bereits erwähnt, ist es am energieeffizientesten, wenn die Hubbewegung genau dann startet, wenn das Fahrwerk anfängt zu bremsen. Hier müssen wiederum mehrere Fälle unterschieden werden. Im einfachsten Fall wird die eingestellte Geschwindigkeit nicht erreicht, z. B. aufgrund eines kleinen zurückzulegenden Weges. Dann startet die Hubbewegung bei der Hälfte der Fahrzeit bzw. des Fahrwegs. Wird die eingestellte Geschwindigkeit jedoch erreicht, sollte die Hubbewegung nach der Phase der konstanten Geschwindigkeit einsetzen. Dieser Weg entspricht, wie Formel $1 \mathrm{zu}$ entnehmen ist, der Summe aus dem Anlaufweg ( $\mathrm{s}_{\mathrm{FW}}$ an $)$ und dem Weg der konstanten Geschwindigkeit ( $\mathrm{S}_{\mathrm{FW}} \mathrm{v}$ konst) bzw. der Differenz aus dem Gesamtweg $\left(\mathrm{S}_{\mathrm{FW} \text { ges }}\right)$ und dem Bremsweg $\left(\mathrm{S}_{\mathrm{FW} \mathrm{Br}}\right)$. Eine solche Bewegung zeigt Abbildung 8.

$\mathrm{S}_{\text {Start }}=\mathrm{S}_{\mathrm{FW} \mathrm{An}}+\mathrm{S}_{\mathrm{FW} \mathrm{v} \mathrm{konst}}=\mathrm{S}_{\mathrm{FW} \mathrm{ges}}-\mathrm{S}_{\mathrm{FW} \mathrm{Br}}$

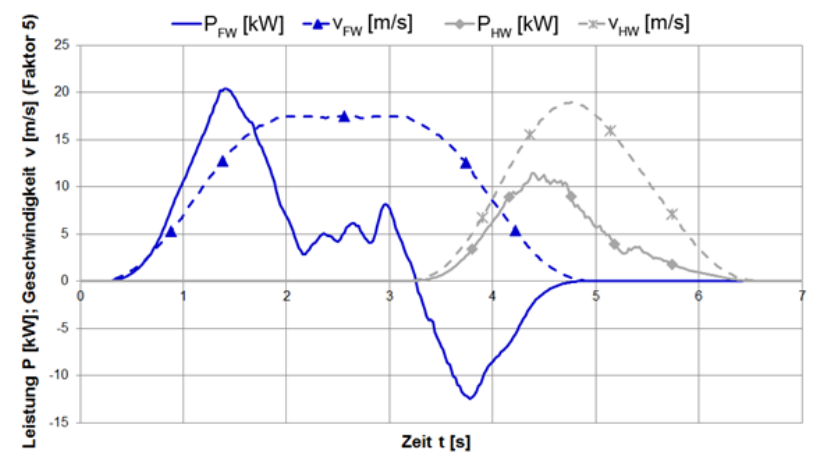

Abbildung 8. Bewegung mit energieoptimalem Start des Hubwerkes

Das Rechenbeispiel für den Transport einer leeren Box, auf dem Abbildung 8 basiert, wird nachfolgend dargelegt. Beim maximalen Fahrweg von 10,0 m werden eine Geschwindigkeit von $3,5 \mathrm{~m} / \mathrm{s}$ und eine Beschleunigung von $3,0 \mathrm{~m} / \mathrm{s}^{2}$ gewählt. Die Fahrzeit beträgt 4,58 s. Es wird ein Hubweg von $5,89 \mathrm{~m}$ mit einer Hubgeschwindigkeit von $4,0 \mathrm{~m} / \mathrm{s}$ überwunden. Das Besondere am ILM-AKL ist, dass die Beschleunigungen des Hubwerkes beim Anfahren und Abbremsen unterschiedlich sind. Im Beispiel beträgt die Beschleunigung 4,0 $\mathrm{m} / \mathrm{s}^{2}$ beim Anfahren und $3,2 \mathrm{~m} / \mathrm{s}^{2}$ beim Abbremsen. Die Hubzeit entspricht 3,24 s.

Der Anfahr- und Bremsweg des Fahrwerkes beträgt 3,07 m. Der Weg der konstanten Geschwindigkeit entspricht beim Fahrwerk 3,86 m. Das Hubwerk sollte nach $6,93 \mathrm{~m}$ Fahrweg (69,3\%) starten. Die Arbeitsspielzeit verlängert sich von $4,58 \mathrm{~s}$ bei einem gleichzeitigen Start der
Antriebe auf 6,20 s. Dies entspricht einer Erhöhung um $35,4 \%$. Allerdings können somit ca. 4,03 kWs, die während der Bremsphase des Fahrwerkes frei werden, für den Hubvorgang genutzt werden. Insgesamt werden bei dieser Bewegung vom Hubwerk ca. 13,80 kWs Elektroenergie aufgenommen. Es kann je nach Bewegungsvorgang jedoch auch vorkommen, dass das Hubwerk nicht die gesamte beim Bremsen des Fahrwerkes frei werdende Energie für das Heben benötigt. In diesem Fall wird die Differenz in das Netz gespeist. Im gewählten Beispiel werden in der Bremsphase des Fahrwerkes 8,71 kWs frei. Folglich müssen $4,68 \mathrm{kWs}$ in das Netz gespeist werden. Der Gesamtenergiebedarf laut Messung unterscheidet sich nicht von einer Bewegung, in der beide Antriebe gleichzeitig starten.

\subsection{STARTPUNKT DES HUBWERKES IM DURCHSATZOPTIMIERTEN BETRIEB}

Unter der Voraussetzung, dass sich durch eine ,energieeffiziente“ Bewegung die Durchsatzleistung nicht verringern darf, kann der Startpunkt so gewählt werden, dass sich die gesamte Arbeitsspielzeit nicht verlängert. Ist die Hubzeit geringer als die Fahrzeit, wird diese von der gesamten Fahrzeit abgezogen, um zu ermitteln, in welcher Phase der Fahrbewegung die Hubbewegung starten soll. Die verschiedenen Phasen sind zur Veranschaulichung in Abbildung 9 dargestellt. Ist die Hubzeit geringer als die Bremszeit des Fahrwerkes (Phase V-VII), d. h. es gilt $\mathrm{t}_{\mathrm{HW} \mathrm{ges}}<\mathrm{t}_{\mathrm{FW} \mathrm{Br}}$, dann startet die Hubbewegung, wie im vorigen Fall, mit Abschluss der Phase der konstanten Geschwindigkeit bzw. falls die eingestellte Geschwindigkeit nicht erreicht wird, nach der Anlaufphase (vgl. Formel 1), da zu Beginn der Bremsphase mehr Energie zurückgespeist wird als zum Ende.

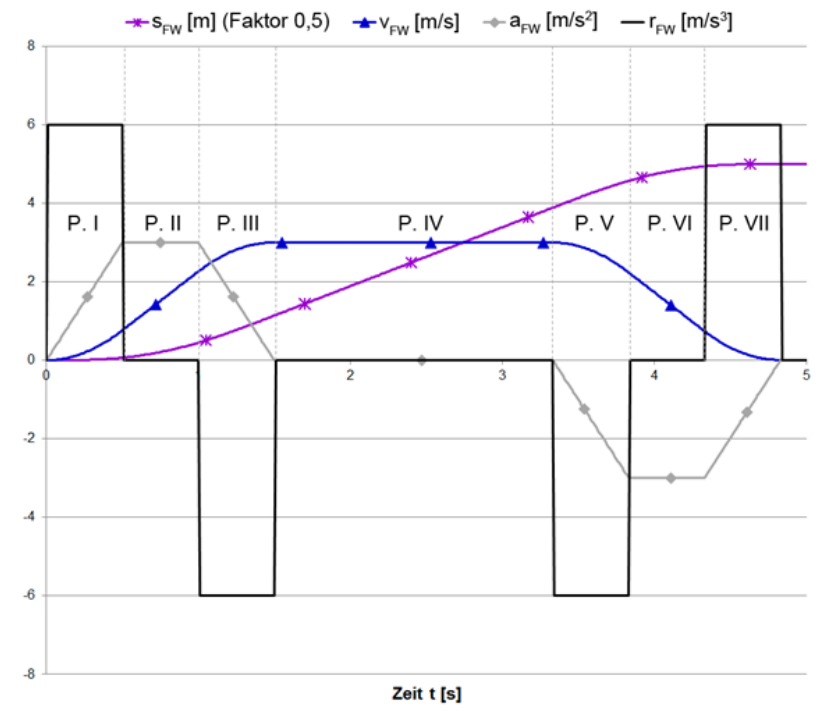

Abbildung 9. Idealisierter Verlauf einer Fahrbewegung

Ist die Hubzeit länger als die Bremszeit des Fahrwerkes, wird das Hubwerk so gestartet, dass beide Triebwerke gleichzeitig an den Zielkoordinaten ankommen. Hier 
muss nach verschiedenen Fällen unterschieden werden, je nachdem in welcher Phase der Fahrbewegung das Hubwerk gestartet werden soll. Soll der Start des Hubwerkes in der Phase der konstanten Geschwindigkeit des Fahrwerkes einsetzen, d. h. es gilt $\mathrm{t}_{\mathrm{FW} \mathrm{Br}}<\mathrm{t}_{\mathrm{HW} \text { ges }}<\mathrm{t}_{\mathrm{FW} \mathrm{Br}}+$ $t_{\mathrm{FW} \text { v konst }}$ (Phase IV), ergibt sich der Fahrweg, bei dem der Hubvorgang gestartet werden soll, nach Formel 2, wobei $\mathrm{V}_{\mathrm{FW} \text { max }}$ die maximal eingestellte (und erreichte) Fahrgeschwindigkeit ist.

$\mathrm{S}_{\mathrm{Start}}=\mathrm{S}_{\mathrm{FW} \mathrm{ges}}-\mathrm{S}_{\mathrm{FW} \mathrm{Br}}-\mathrm{V}_{\mathrm{FW} \mathrm{max}} \cdot\left(\mathrm{t}_{\mathrm{HW} \mathrm{ges}}-\mathrm{t}_{\mathrm{FW} \mathrm{Br}}\right)$

Ist die Hubzeit größer als die Summe aus der Bremszeit und der Zeit der konstanten Geschwindigkeit des Fahrwerkes, sollte der Start des Hubwerkes in der Anfahrphase des Fahrwerkes erfolgen. Um den exakten Startpunkt zu ermitteln, muss wieder berechnet werden, in welcher Phase der Fahrwerksbeschleunigung das Hubwerk starten soll. Hier ist zu unterscheiden, ob die Beschleunigung des Fahrwerkes zunimmt $(r>0$, Phase I), konstant ist $(\mathrm{r}=0$, Phase II) oder abnimmt $(\mathrm{r}<0$, Phase III). Nimmt die Beschleunigung ab (Phase III), d. h. es gilt $\mathrm{t}_{\mathrm{FW} \mathrm{Br}}+\mathrm{t}_{\mathrm{FW} \mathrm{v} \mathrm{konst}}<\mathrm{t}_{\mathrm{HW} \text { ges }}<\mathrm{t}_{\mathrm{FW} \mathrm{Br}}+\mathrm{t}_{\mathrm{FW} \text { konst }}+\mathrm{t}_{\mathrm{FW} \text { a err }}$, wird der Fahrweg, nach dem das Hubwerk gestartet werden soll, nach Formel 3 berechnet. Dabei entspricht $t_{\mathrm{FW}}$ a err der notwendigen Zeit, um die maximal erreichte Beschleunigung auf $0,0 \mathrm{~m} / \mathrm{s}^{2}$ zu reduzieren bzw. die Zeit bis sich die maximale Beschleunigung einstellt.

$$
\begin{aligned}
\mathrm{s}_{\mathrm{Start}}= & -\frac{1}{6} \cdot \mathrm{r}_{\mathrm{FW}} \cdot\left(\mathrm{t}_{\mathrm{FW} \mathrm{ges}}-\mathrm{t}_{\mathrm{HW} \mathrm{ges}}-\frac{\mathrm{v}_{\mathrm{FW} \mathrm{err}}}{\mathrm{a}_{\mathrm{FW} \mathrm{err}}}\right)^{3} \\
& +\frac{\mathrm{a}_{\mathrm{FW} \mathrm{err}}}{2} \cdot\left(\mathrm{t}_{\mathrm{FW} \mathrm{ges}}-\mathrm{t}_{\mathrm{HW} \mathrm{ges}}-\frac{\mathrm{v}_{\mathrm{FW} \mathrm{err}}}{\mathrm{a}_{\mathrm{FW} \mathrm{err}}}\right)^{2} \\
& +\left(\mathrm{v}_{\mathrm{FW} \mathrm{err}}-\frac{\mathrm{a}_{\mathrm{FW} \mathrm{err}}}{2 \cdot \mathrm{r}_{\mathrm{FW}}}\right) \cdot\left(\mathrm{t}_{\mathrm{FW} \mathrm{ges}}-\mathrm{t}_{\mathrm{HW} \mathrm{ges}}-\frac{\mathrm{v}_{\mathrm{FW} \mathrm{err}}}{\mathrm{a}_{\mathrm{FW} \mathrm{err}}}\right) \\
& +\frac{\mathrm{v}_{\mathrm{FW} \mathrm{err}}}{2 \cdot \mathrm{a}_{\mathrm{FW} \mathrm{err}}}-\frac{\mathrm{a}_{\mathrm{FW} \mathrm{err}} \cdot \mathrm{v}_{\mathrm{FW} \mathrm{err}}}{2 \cdot \mathrm{r}_{\mathrm{FW}}}+\frac{\mathrm{a}_{\mathrm{FW} \mathrm{err}}}{6 \cdot \mathrm{r}_{\mathrm{FW}}}
\end{aligned}
$$

Ist die Beschleunigung des Fahrwerkes zum Startzeitpunkt konstant (Phase II), d. h. es gilt $\mathrm{t}_{\mathrm{FW} \mathrm{Br}}+\mathrm{t}_{\mathrm{FW} \mathrm{v} \text { konst }}$ $+t_{\mathrm{FW} \mathrm{aerr}}<\mathrm{t}_{\mathrm{HW} \mathrm{ges}}<\mathrm{t}_{\mathrm{FW} \text { ges }}-\mathrm{t}_{\mathrm{FW} \text { a err }}$, wird der Startweg mit Formel 4 bestimmt.

$$
\begin{aligned}
\mathrm{S}_{\mathrm{Start}}= & \frac{\mathrm{a}_{\mathrm{FW} \mathrm{err}}}{2} \cdot\left(\mathrm{t}_{\mathrm{FW} \mathrm{ges}}-\mathrm{t}_{\mathrm{HW} \mathrm{ges}}-\frac{\mathrm{a}_{\mathrm{FW} \mathrm{err}}}{\mathrm{r}_{\mathrm{FW}}}\right)^{2} \\
& +\frac{\mathrm{a}_{\mathrm{FW} \mathrm{err}}}{2 \cdot \mathrm{r}_{\mathrm{FW}}} \cdot\left(\mathrm{t}_{\mathrm{FW} \mathrm{ges}}-\mathrm{t}_{\mathrm{HW} \mathrm{ges}}-\frac{\mathrm{a}_{\mathrm{FW} \mathrm{err}}}{\mathrm{r}_{\mathrm{FW}}}\right)+\frac{\mathrm{a}_{\mathrm{FW} \mathrm{err}}{ }^{3}}{6 \cdot \mathrm{r}_{\mathrm{FW}}{ }^{2}}
\end{aligned}
$$

Befindet sich der Startpunkt in der Phase, in der die Beschleunigung zunimmt (Phase I), d. h. es gilt $t_{\mathrm{FW} \text { ges }}$ $\mathrm{t}_{\mathrm{FW} \text { a err }}<\mathrm{t}_{\mathrm{HW} \text { ges }}<\mathrm{t}_{\mathrm{FW} \text { ges }}$, ergibt sich der Startweg aus Formel 5.

$\mathrm{S}_{\mathrm{Start}}=\mathrm{r}_{\mathrm{FW}} / 6 \cdot\left(\mathrm{t}_{\mathrm{FW} \mathrm{ges}}-\mathrm{t}_{\mathrm{HW} \mathrm{ges}}\right)^{3}$
Durch die Optimierung des Startpunktes des Hubwerkes unter der Beschränkung, dass sich die Durchsatzleistung nicht verringern darf, wird dem Hubwerk trotzdem möglichst viel Bremsenergie des Fahrwerkes für den Hubvorgang zur Verfügung gestellt.

Der Startzeitpunkt im durchsatzoptimierten Betrieb wird zum besseren Verständnis an einem Beispiel berechnet. Wieder soll das Beispiel Abschnitt 6.1 herangezogen werden $\left(\mathrm{s}_{\mathrm{FW} \text { ges }}=10,0 \mathrm{~m}\right.$ und $\left.\mathrm{s}_{\mathrm{HW} \text { ges }}=5,89 \mathrm{~m}\right)$. Für dieses Beispiel gilt Formel 3. Die Hubzeit beträgt 3,24 s und die Fahrzeit 4,58 s. Somit liegt der Startpunkt des Hubwerkes bei 1,81 m (18,1\%) Fahrweg. Beim Bremsen des Fahrwerkes werden in Summe 8,71 kWs frei, wovon für die Hubbewegung 3,17 kWs genutzt werden können. Somit werden $5,54 \mathrm{kWs}$ in das Netz gespeist. Insgesamt werden vom Hubwerk 13,80 kWs aufgenommen.

\subsection{OPTIMALE GESCHWINDIGKEIT DES FAHRWERKES IM DURCHSATZOPTIMIERTEN BETRIEB}

Darf sich die Durchsatzleistung nicht verschlechtern und die Hubzeit ist länger als die Fahrzeit, d. h. es gilt $t_{\mathrm{FW} \mathrm{ges}}<\mathrm{t}_{\mathrm{HW} \text { ges }}$, kann die Geschwindigkeit des Fahrwerkes so weit reduziert werden, dass Fahr- und Hubwerk gleichzeitig bei den Zielkoordinaten ankommen. Für die Berechnung wird Formel 6 herangezogen. Durch Einsetzen ergibt sich die optimal reduzierte Fahrgeschwindigkeit $\left(\mathrm{v}_{\mathrm{FW} \text { red }}\right.$ ) aus Formel 7. Hier ist nun zuerst zu unterscheiden, ob die Summe aus Formel 7 größer oder kleiner ist als die maximal eingestellte Geschwindigkeit ( $\left.\mathrm{v}_{\mathrm{FW} \text { max }}\right)$. Ist das Ergebnis größer als $\mathrm{v}_{\mathrm{FW} \text { max }}$, entspricht $\mathrm{v}_{\mathrm{FW} \text { red }}$ der Differenz mit der Wurzel in Formel 7, sonst der Summe (vgl. Beispiel S. 8).

$t_{\mathrm{HW} \mathrm{ges}}=t_{\mathrm{FW} \mathrm{ges}}=t_{\mathrm{FW} \mathrm{An}}+t_{\mathrm{FW} \mathrm{v} \mathrm{konst}}+\mathrm{t}_{\mathrm{FW} \mathrm{Br}}$
$\mathrm{V}_{\mathrm{FW} \mathrm{red} 1,2}=-\frac{1}{2} \cdot\left(\frac{\mathrm{a}_{\mathrm{FW} \mathrm{err}}{ }^{2}}{\mathrm{r}_{\mathrm{FW}}}-\mathrm{t}_{\mathrm{HW} \mathrm{ges}} \cdot \mathrm{a}_{\mathrm{FW} \mathrm{err}}\right)$

$\pm \sqrt{\left[-\frac{1}{2} \cdot\left(\frac{\mathrm{a}_{\mathrm{FW} \mathrm{err}}{ }^{2}}{\mathrm{r}_{\mathrm{FW}}}-\mathrm{t}_{\mathrm{HW} \mathrm{ges}} \cdot \mathrm{a}_{\mathrm{FW} \mathrm{err}}\right)\right]^{2}-\mathrm{s}_{\mathrm{FW} \mathrm{ges}} \cdot \mathrm{a}_{\mathrm{FW} \mathrm{err}}}$

Zweitens ist zu überprüfen, ob die berechnete reduzierte Geschwindigkeit ( $\mathrm{v}_{\mathrm{FW} \text { red }}$ ) unter der Grenzgeschwindigkeit liegt, die sich beim Erreichen der vorgegebenen Beschleunigung einstellt. Die Grenzgeschwindigkeit ist die Geschwindigkeit, unter der die eingestellte Beschleunigung nicht mehr erreicht wird $\left(\mathrm{a}_{\mathrm{FW} \mathrm{err}} / \mathrm{r}_{\mathrm{FW}}\right)$ und somit die Phase der konstanten Beschleunigung (Phase II und Phase VI) entfällt. Ist $\mathrm{V}_{\mathrm{FW}}$ red größer als die Grenzgeschwindigkeit, gilt $a_{\mathrm{FW} \mathrm{err}}=\mathrm{a}_{\mathrm{FW} \text { max }}$. Ist $\mathrm{v}_{\mathrm{FW} \text { red }}$ kleiner als die Grenzgeschwindigkeit, z. B. weil der Fahrweg sehr kurz ist, wird die gewählte Beschleunigung nicht erreicht. In diesem Fall existiert weder eine Phase mit konstanter Geschwindigkeit (Phase VI), noch mit konstanter Beschleunigung (Phase II und Phase VI). Die erreichte Beschleunigung $\left(\mathrm{a}_{\mathrm{FW}}\right.$ err $)$ ergibt sich dann aus Formel 8. 
$\mathrm{a}_{\mathrm{FW} \mathrm{err}}=\frac{\mathrm{r}_{\mathrm{FW}}}{2} \cdot \mathrm{t}_{\mathrm{FW} \mathrm{An}}=\frac{\mathrm{r}_{\mathrm{FW}}}{2} \cdot \sqrt[3]{\frac{4 \cdot \mathrm{s}_{\mathrm{FW} \mathrm{ges}}}{\mathrm{r}_{\mathrm{FW}}}}$

Ein Sonderfall ist zu beachten, wenn die ermittelte reduzierte Geschwindigkeit ( $\mathrm{v}_{\mathrm{FW} \text { red }}$ ) so gering ist, dass die eingestellte Beschleunigung nicht erreicht wird. Hier entfällt die Phase mit konstanter Geschwindigkeit nicht und es gilt $\mathrm{a}_{\mathrm{FW} \text { max }}>\sqrt{\mathrm{v}_{\mathrm{FW} \text { red }} \cdot \mathrm{r}_{\mathrm{FW}}}$. Somit wird die erreichte Beschleunigung reduziert. Die Folge ist, dass die Phase der konstanten Beschleunigung eliminiert wird. Durch diese Vorgehensweise können die Parameter der optimalen Geschwindigkeit und Beschleunigung jedoch nur näherungsweise bestimmt werden, sodass die Fahrzeit noch leicht unter der Hubzeit liegt (durch das Fehlen der Phase mit konstanter Beschleunigung). Die sich einstellende Beschleunigung entspricht Formel 9.

$\mathrm{a}_{\mathrm{FW} \mathrm{err}}=\sqrt{\mathrm{v}_{\mathrm{FW} \mathrm{red}} \cdot \mathrm{r}_{\mathrm{FW}}}$

Als Anschauungsbeispiel soll eine Bewegung mit einem Fahrweg von 4,00 m, einer Fahrgeschwindigkeit von eingestellten $4,0 \mathrm{~m} / \mathrm{s}$ sowie einer Beschleunigung von $3,0 \mathrm{~m} / \mathrm{s}^{2}$ und $6,20 \mathrm{~m}$ Hubweg mit 4,0 m/s Hubgeschwindigkeit und $4,0 \mathrm{~m} / \mathrm{s}^{2}$ beim Beschleunigen bzw. $3,2 \mathrm{~m} / \mathrm{s}^{2}$ beim Verzögern des Hubwerkes ausgeführt werden. Die Hubzeit entspricht bei diesen Werten 3,30 s und die Fahrzeit 2,88 s. Es wird vom Fahrwerk allerdings nur eine Geschwindigkeit von $2,91 \mathrm{~m} / \mathrm{s}$ erreicht. Diese kann aber aufgrund der geringeren Fahrzeit im Vergleich zur Hubzeit weiter reduziert werden. Für die Ermittlung der reduzierten Geschwindigkeit wird Formel 7 herangezogen. Die Summe mit der Wurzel in Formel 7 würde zu einer höheren Geschwindigkeit als der eingestellten führen. Daher liefert die Differenz mit der Wurzel aus Formel 7 das Ergebnis. Die reduzierte Geschwindigkeit sollte in diesem Beispiel 1,83 m/s betragen, damit beide Antriebe zeitgleich bei den Zielkoordinaten ankommen und der Energiebedarf so weit wie möglich reduziert wird. In diesem Beispiel werden bei der eingestellten Geschwindigkeit von 4,0 m/s durchschnittlich 11,71 kWs Elektroenergie vom Fahrwerk aufgenommen und 6,90 kWs Elektroenergie vom Fahrwerk zurückgespeist. Bei der auf $1,83 \mathrm{~m} / \mathrm{s}$ reduzierten Geschwindigkeit werden $6,22 \mathrm{kWs}$ aufgenommen und 2,33 kWs werden beim Bremsvorgang des Fahrwerkes frei. Der Gesamtelektroenergiebedarf sinkt also um 0,92 kWs $(19,1 \%)$, wenn die Geschwindigkeit weiter reduziert wird. Die Arbeitsspielzeit verlängert sich dagegen nicht, weil die Hubzeit maßgebend ist.

Wird anstatt der Geschwindigkeit die Beschleunigung des Fahrwerkes so weit reduziert, dass Fahr- und Hubwerk gleichzeitig am Ziel ankommen, beträgt die Energieaufnahme des Fahrwerkes 9,61 kWs und es werden 5,07 kWs beim Bremsen frei. Der Gesamtenergiebedarf ist also mit 4,54 kWs geringer als bei vorgegebenen Geschwindigkeit von 4,0 m/s, aber höher als der Energiebedarf des Fahrwerkes bei optimierter Geschwindigkeit
$(1,83 \mathrm{~m} / \mathrm{s})$. Dieses Ergebnis wurde auch für kleinere Wege getestet und resultiert aus den unterschiedlichen Verläufen der Leistungs- und Geschwindigkeitskurven (siehe Abbildung 10). Bis sich die Geschwindigkeiten angleichen, ist bei der kleineren Beschleunigung die Leistungsaufnahme des Fahrwerkes geringer. Sobald diese Geschwindigkeit jedoch erreicht ist, wird das Fahrwerk weiter beschleunigt. $\mathrm{Ab}$ hier ist die Leistungsaufnahme $\mathrm{u}$. a. durch das höhere Widerstandsmoment größer. Dies kann auch nicht durch die höhere beim Bremsen frei werdende Energie kompensiert werden.

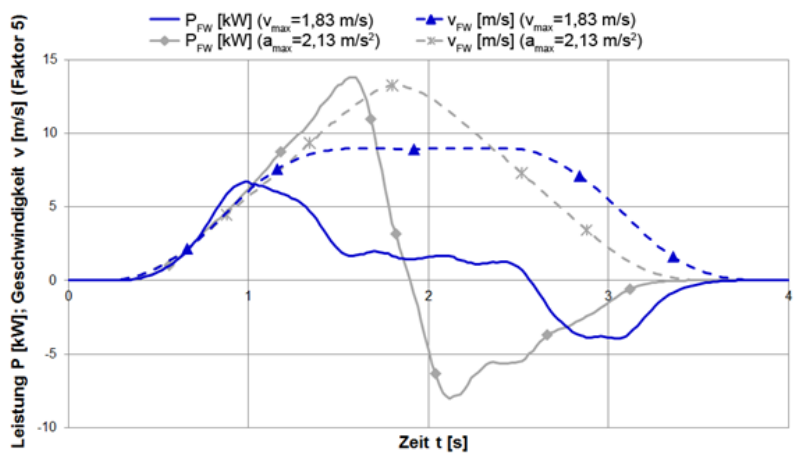

Abbildung 10. Vergleich einer Fahrt mit reduzierter Geschwindigkeit und reduzierter Beschleunigung

\section{DER EINFLUSS DER NUTZMASSE AUF DEN ENERGIEBEDARF}

Weil nicht nur die Geschwindigkeiten und Beschleunigungen Einfluss auf den Energiebedarf einer RBGBewegung haben, sondern auch die Nutzmasse, soll an dieser Stelle deren Einfluss aufgezeigt werden. Am ILMAKL ist die Nutzmasse durch die maximale Fachlast auf $30 \mathrm{~kg}$ begrenzt, obwohl die Traglast des RBG bis zu $100 \mathrm{~kg}$ beträgt. Die Masse hat theoretisch Einfluss auf den Energiebedarf des Fahr- und Hubwerkes $\left(E_{\mathrm{kin}}=1 / 2 \cdot \mathrm{m} \cdot \mathrm{v}^{2}\right.$ und $\mathrm{E}_{\mathrm{pot}}=\mathrm{m} \cdot \mathrm{g} \cdot \Delta \mathrm{h}$ ), allerdings sorgt die hohe Eigenmasse des RBG im Vergleich zur maximalen Nutzmasse $(1.996 \mathrm{~kg}$ vs. $30 \mathrm{~kg}$ ) dafür, dass der Einfluss der Nutzmasse auf den Energiebedarf des Fahrwerkes verschwindend gering ist. Diese Aussage ist jedoch nicht bei Paletten-RBGs zutreffend, weil bei diesen höhere Massen transportiert werden und das Eigenmasse-Nutzmasse-Verhältnis dementsprechend besser ist.

An dieser Stelle wird daher nur der Einfluss der Nutzmasse auf die Energieaufnahme und -rückspeisung des Hubwerkes aufgezeigt. Da die Aufnahme und Rückspeisung, wie in Abschnitt 5 beschrieben, lediglich von der Masse und dem zurückzulegenden Hub- bzw. Senkweg abhängen, liegen die Werte für die Energieaufnahme und -rückspeisung bei einer höhren Nutzmasse immer über den Werten mit geringerer Nutzmasse. In Abbildung 11 werden die Energiewerte des Hubwerkes bei einer transportierten Nutzmasse von $0 \mathrm{~kg}$ und $30 \mathrm{~kg}$ gegenübergestellt. 
Die transportierte Nutzmasse kann aufgrund der vorgegebenen Transportaufträge zwar nicht beeinflusst werden, aber der Lagerort eines einzulagernden Behälters kann festgelegt werden. Daher wird bei der Betrachtung des Energiebedarfs empfohlen, dass in einem AKL (wenn die Nutzlast bekannt ist) Behälter mit höheren Nutzlasten in den Ebenen nahe der Ebene des Einlagerungspunktes eingelagert werden sollten. Bei Palettenhochregallagern sollten, wenn die Masse auf den Paletten bekannt ist, Paletten mit höheren Nutzlasten zusätzlich möglichst nahe des Ein- und Auslagerpunktes eingelagert werden.

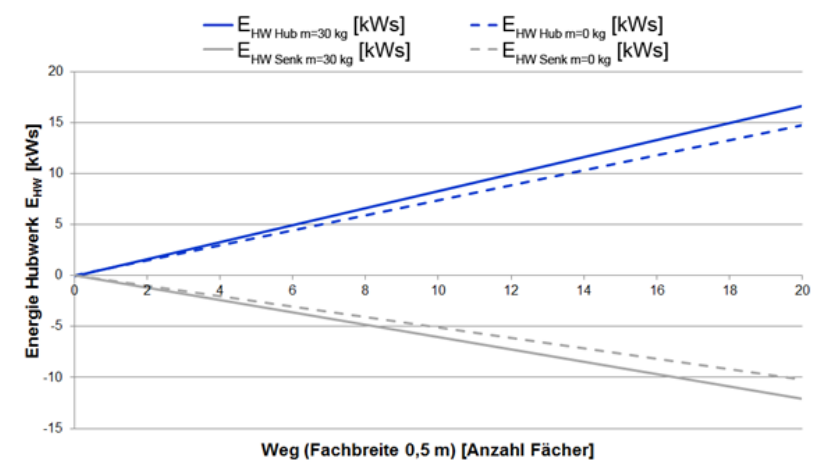

Abbildung 11. Vergleich der Energiewerte des Hubwerkes bei $0 \mathrm{~kg}$ und $30 \mathrm{~kg}$ Nutzmasse

\section{DER ENERGIEBEDARF VON EINZEL- UND DOPPELSPIELEN}

Leerfahrten sind ineffizient und führen somit zu einem höheren Energiebedarf pro transportierter Mengeneinheit. Folglich sollen an dieser Stelle die Energieeffizienz von Einzel- und Doppelspielen anhand der Referenzspiele der FEM-Richtlinie 9.851 bzw. der VDI-Richtlinie 3.561 am Beispiel des ILM-AKL bestimmt werden.

Für fest vorgegebene Parameter können für die Regalfläche die sogenannten isoenergetischen Fächer bestimmt werden, also die Fächer, die mit dem gleichen Gesamtenergiebedarf $\mathrm{zu}$ erreichen sind [Sch12, S. 31]. Da sich je nach Einstellung der Geschwindigkeiten und Beschleunigungen die Energiebedarfe ändern, verschiebt sich ggf. auch die Lage der isoenergetischen Fächer. Beim Hubwerk ist lediglich zwischen einer Hub- und Senkbewegung zu unterscheiden, weil der Energiebedarf des Hubwerkes, wie in Abschnitt 5 erläutert, unabhängig von der Geschwindigkeit und der Beschleunigung ist. Abbildung 12 zeigt die isoenergetischen Fächer einer Hubbewegung für die gegebenen Parameter. Die isoenergetischen Fächer für eine Senkbewegung zeigt Abbildung 13.

Die Referenzpunkte laut FEM-Richtlinie sind $\mathrm{P}_{1}=\mathrm{X} 16 \mathrm{Y} 15$ und $\mathrm{P}_{2}=\mathrm{X} 07 \mathrm{Y} 05$ (vgl. Abbildung 12) [FEM9851, S.9], nach der VDI-Richtlinie ergibt sich $\mathrm{P}_{1}=\mathrm{X} 07 \mathrm{Y} 05$ und $\mathrm{P}_{2}=\mathrm{X} 17 \mathrm{Y} 14$ (vgl. Abbildung 13) [VDI3561, S. 4]. Diese Punkte werden vom Ein-und Auslagerpunkt (X21Y02) angefahren. Die Versuche werden mit für RBGs typischen Parametern durchgeführt: Geschwindigkeit Fahrwerk $\left(\mathrm{v}_{\mathrm{FW}}\right) 4,0 \mathrm{~m} / \mathrm{s}$, Beschleunigung Fahrwerk ( $\left.\mathrm{a}_{\mathrm{FW}}\right) 3,0 \mathrm{~m} / \mathrm{s}^{2}$, Geschwindigkeit Hubwerk $\left(\mathrm{v}_{\mathrm{HW}}\right)$ $3,0 \mathrm{~m} / \mathrm{s}$, Beschleunigung Hubwerk $\left(\mathrm{a}_{\mathrm{HW}}\right) \quad 3,0 \mathrm{~m} / \mathrm{s}^{2}$ [VDI3630, S. 5].

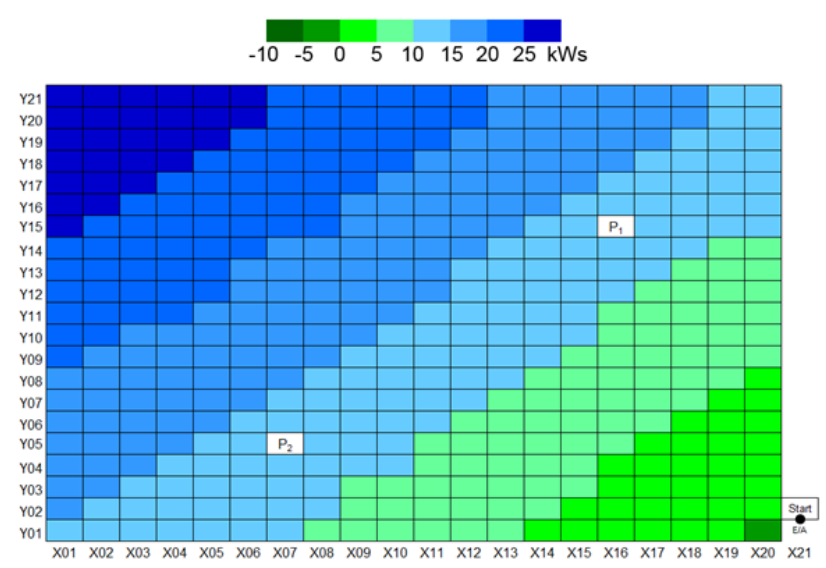

Abbildung 12. Isoenergetische Fächer einer Hubbewegung bei einer Fahrgeschwindigkeit von 4,0 m/s mit Referenzfächern nach FEM 9.851

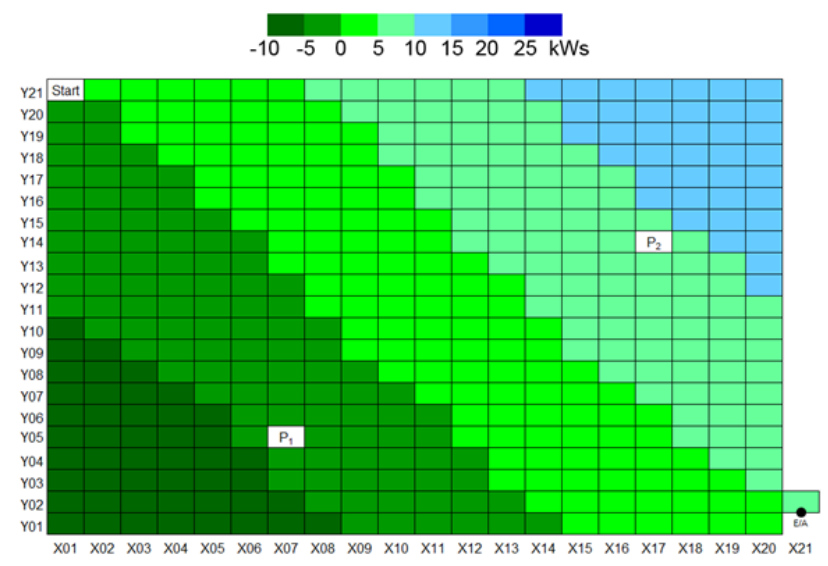

Abbildung 13. Isoenergetische Fächer einer Senkbewegung bei einer Fahrgeschwindigkeit von 4,0 m/s mit Referenzfächern nach VDI 3.561

Tabelle 4 zeigt die auf Doppelspiele normierten Gesamtelektroenergiebedarfe der Einzel- und Doppelspiele (ES und DS) sowie die mittleren Einzel- und Doppelspielzeiten für die Referenzspiele der FEM- und VDI-Richtlinie. Wie in Tabelle 4 ersichtlich wird, sinkt durch Doppelspiele nicht nur die mittlere Arbeitsspielzeit, sondern auch (überproportional) der durchschnittliche Energiebedarf pro Behälter, weil bei Doppelspielen die zurückzulegenden Wege verringert werden. Der Durchsatz für FEMund VDI-Einzelspiele beträgt beim ILM-AKL theoretisch rund 216 Behälter pro Stunde. Beim Einsatz von Doppelspielen sind bis zu 243 Behälter/ h möglich. Diese hohen Durchsatzzahlen sind das Resultat der geringen Maße des ILM-AKL. 
Tabelle 4. Gesamtelektroenergiebedarfe und Spielzeiten pro Behälter für FEM- und VDI-Referenzspiele

\begin{tabular}{|c|c|c|c|c|}
\hline Spiel & $\boldsymbol{\varnothing E}[\mathbf{k W s / B . ]}$ & $\boldsymbol{\emptyset t}[\mathbf{s} / \mathbf{B .}]$ & $\boldsymbol{\Delta E}[\mathbf{\%}]$ & $\boldsymbol{\Delta t}[\mathbf{\%}]$ \\
\hline FEM-ES & 16,33 & 16,66 & 0,0 & 0,0 \\
\hline FEM-DS & 13,30 & 14,81 & $-18,6$ & $-11,1$ \\
\hline VDI-ES & 15,38 & 16,48 & 0,0 & 0,0 \\
\hline VDI-DS & 12,10 & 14,79 & $-21,3$ & $-10,3$ \\
\hline
\end{tabular}

\section{ZuSAMmenfassung Und AUSBlick}

In diesem Beitrag wurde der Einfluss verschiedener kinematischer Parameter auf den Energiebedarf (von Einzelfahrten) eines RBG aufgezeigt. Es wurde nachgewiesen, dass verringerte Geschwindigkeiten und Beschleunigungen den Energiebedarf des Fahrwerkes verringern. Auf das Hubwerk hat die Reduzierung von Geschwindigkeit und Beschleunigung keinen Einfluss. Eine Veränderung dieser Parameter ist beim Hubwerk nur zu empfehlen, um den Verschleiß zu reduzieren - aus energetischer Sicht ist eine Variation von Geschwindigkeit und Beschleunigung jedoch nicht sinnvoll.

Verringerte Geschwindigkeiten und Beschleunigungen führen zu höheren Fahr- und Hubzeiten. Dadurch kann sich gegebenenfalls die Arbeitsspielzeit erhöhen. Eine Reduzierung der Parameter ist daher nur zu empfehlen, wenn es die geforderte Durchsatzleistung zulässt. Dann kann auch der Startpunkt des Hubwerkes energetisch optimiert werden. Kann die Durchsatzleistung nicht verringert werden, ist der Startpunkt der Hubbewegung so einzustellen, dass beide Antriebe gleichzeitig bei den Zielkoordinaten ankommen. Somit kann trotzdem möglichst viel Energie, die beim Bremsen des Fahrwerkes frei wird, für den Hubvorgang genutzt werden. Bei einer Senkbewegung sollten beide Antriebe gleichzeitig starten um die potentielle, frei werdende Energie des Hubwerkes für die Beschleunigung des Fahrwerkes maximal ausnutzen zu können.

Können die Parameter aufgrund eines hohen geforderten Durchsatzes nicht reduziert werden und die Hubzeit ist größer als die Fahrzeit, ist es möglich, die Fahrgeschwindigkeit so einzustellen, dass Fahr- und Hubwerk gleichzeitig am Ziel ankommen. So wird trotz einer hohen geforderten Durchsatzleistung der Energiebedarf verringert. Eine Beschleunigungsreduktion ist bei gleicher Fahrzeit nicht sinnvoll, weil durch die höhere erreichte Geschwindigkeit insgesamt mehr Energie aufgewendet werden muss als bei einer Fahrt mit verringerter Geschwindigkeit.

Auch die Nutzmasse hat einen Einfluss auf den Energiebedarf eines RBG. Zwar kann die transportierte Nutzmasse aufgrund der vorgegebenen Transportaufträge nicht beeinflusst werden, aber bei der Einlagerung kann der Lagerort vorgegeben werden. Deshalb sollten in einem AKL nach Möglichkeit, falls die zum Zeitpunkt des Transportes gegebene Nutzmasse eines Behälters bekannt ist, die Behälter mit höheren Nutzlasten in den Ebenen nahe der Ebene des Einlagerungspunktes eingelagert werden. In Palettenhochregalen sollten Paletten mit höheren Nutzmassen zusätzlich nahe des Ein- und Auslagerpunktes eingelagert werden.

Wie allgemein bekannt, wird durch Doppelspiele die durchschnittliche Spielzeit verringert und so die Durchsatzleistung erhöht. Durch die Reduzierung der zurückzulegenden Wege verringert sich weiterhin der durchschnittliche Energiebedarf pro transportierter Mengeneinheit (z. B. Behälter oder Palette).

Über die in diesem Beitrag beschriebenen Betrachtungen von Einzelbewegungen sollen die durchschnittlichen Energiebedarfe verschiedener Lagerstrategien erforscht und gegebenenfalls energieeffizientere Lagerstrategien definiert werden.

\section{LITERATUR}

[FEM9851] Fédération Européenne de la Manutention (FEM): FEM 9.851 - Leistungsnachweis für Regalbediengeräte - Spielzeiten. Richtlinie, Frankfurt am Main, Verband Deutscher Maschinen und Anlagenbau (VDMA), 2003

[Kra08] Kramm, Matthias: Der Energieausweis für Distributionszentren. In: Tagungsband Energieeffizienz im Lager. Köln, 2008

[Muh11] Muhlfinger, Rudolf: Schwarze Zahlen mit grüner Technik. In: Green Logistics, Sonderbeilage der DVZ - Deutsche Logistik-Zeitung vom 24.05.2011. Hamburg: Deutscher Verkehrs-Verlag, 2011

[Sch12] Schulz, Robert; Monecke, Jörg; Zadek, Hartmut: Isoenergetische Fächer eines Automatischen Kleinteilelagers. In: Wolf-Kluthausen, Hanne (Hrsg.): Jahrbuch Logistik 2012, Korschenbroich: free beratung $\mathrm{GmbH}, 2012$, S. 28-33

[VDI3561] Verein Deutscher Ingenieure (VDI): VDI-Richtlinie 3.561 - Testspiele zum Leistungsvergleich und zur Abnahme von Regalbediengeräten. Richtlinie, Berlin: Beuth Verlag, 1973

[VDI3630] Verein Deutscher Ingenieure (VDI): VDI-Richtlinie 3.630 - Automatische Kleinteilelager. Richtlinie, Berlin: Beuth Verlag, 2006 